\title{
Thoughts on the Politics of COVID-19
}

\author{
Colin A. Ross*
}

Institute for Psychological Trauma, 1701 Gateway, Suite 349, Richardson, TX 75080, USA

*Corresponding author: Colin A. Ross, Institute for Psychological Trauma, 1701 Gateway, Suite 349, Richardson, TX 75080, USA

Received: July 08, 2021; Accepted: July 15, 2021; Published: July 24, 2021

In previous papers in the Journal of Neurology and Neurocritical Care, I have provided a scholarly, referenced, data-based analysis of misinformation provided by the CDC, governments, the NIH, NIAID and leading physicians and medical journals concerning the COVID-19 pandemic [1-6]. I have had two doses of the Pfizer vaccine and am not an anti-vaxxer. Here I will provide some thoughts on the politics and social dynamics of the response to the pandemic.

\section{Comparing COVID-19 to the Flu}

Early in the pandemic, comparison of the SARS-CoV-2 virus to the flu was disallowed. This was peculiar since both are viral respiratory illnesses and both can have systemic complications. The 1918-1919 flu pandemic killed far more people than have died from the current coronavirus pandemic. Comparison of the SARS-CoV-2 virus to the flu virus was equated with minimizing the seriousness of the current pandemic. Why? It was OK to compare the two viruses as long as you were saying that the SARS-CoV-2 virus is far more dangerous. In actual fact, of the two biggest pandemics since the end of World War I, the flu pandemic was the more deadly by far.

\section{Denigrating Hydroxychloroquine and Ivermectin}

Two drugs for treatment of COVID-19 - hydroxychloroquine and ivermectin - have been ridiculed, lambasted and rejected by leading medical journals and the mainstream media. Both are generic, have been in use for decades and have very reasonable side effect profiles. The ridicule began before there was adequate published data to evaluate them. At the same time, remdesivir and convalescent plasma were widely endorsed by leading medical journals in the absence of adequate controlled data either to support or reject their usefulness. Why? Remdesivir and convalescent plasma proved to be either ineffective or only slightly superior to placebo. Yet both got the green light as tools to be used in a desperate situation. This is not balanced science. Backing remdesivir and convalescent plasma while putting down hydroxychloroquine and ivermectin is easy to understand - there's very little money to be made from generic drugs. The duty of the medical profession, apparently, is to denigrate generic drugs while endorsing expensive interventions that are no more effective. This makes medicine look like a sales force for drug companies - not a very good strategy for increasing trust in the medical profession, or for reducing vaccine hesitancy.

\section{Justifying Changes in Face Mask Policies with Non-Existent 'Emerging Data'}

Except in isolation units and operating rooms, hospital staffs have never worn face masks inside hospitals, prior to the onset of the pandemic. The WHO and the US Surgeon General stated in February, 2020 that wearing face masks in public is unnecessary and does not reduce viral transmission. Within a few months, the NIH, NIAID, CDC, Surgeon General and leading medical journals did a 180-degree turn on face masks. This resulted in face masks being widely mandated. The change in policy was justified by the emergence of new data, but in fact no such data existed. In May, 2021 the CDC, governments and some large corporations changed their position on face masks in public, saying that they are not necessary, again justifying the change in policy as being based on new evidence. There was in fact no new evidence. Changes in face mask mandates and policies in both directions were said to be based on science and new data, when no such data existed. Anyone who opposed the policies, in either direction, was accused of being against science.

The claim to be science-based has been used as a propaganda tool and as a tactic to discredit dissenters - now we see that the tool has been used in both directions - to mandate face masks and to remove mandates. Why is this happening? Is the face mask manufacturing lobby that strong? Is the goal social control? Is it a motivational technique to scare people into getting vaccinated? Is it just a power trip? Any, all or none of these motives could be at work. The problem is not with the politicians - they are politicians and are always attacking the opposition.

The problem is the medical profession, which has acted as an agent of the state in contradiction to the science concerning face masks, and has attacked dissenting physicians.

\section{Death Counts and Infection Rates}

It has been very difficult to evaluate what is going on in the pandemic because of false positive and false negative test results. Still, we have to use the best tools available. A more serious problem is the fact that hospitals have been getting paid more if they code a death as a COVID-19 death - even in the absence of a positive test. These problems create suspicion that the severity of the pandemic has been exaggerated by the CDC and the medical profession, which in turn causes distrust and results in vaccine hesitancy. Why should the public believe the CDC and doctors about vaccines when both have been all over the map on face masks and disease rates? Instead of blaming the public, the medical profession should examine how it has contributed to vaccine hesitancy. You can fool some of the people some of the time, but you can't fool all of the people all of the time. 


\section{Whatever Happened To Contact Tracing and Testing?}

Early in the COVID-19 pandemic, the pillars of public health interventions were face masks, vaccines (not yet on the market), social distancing, contact tracing, lockdowns and testing. Contact tracing and testing quietly disappeared from public discussion and recommendations. Why? I don't know, but I assume that it finally became undeniable that contact tracing was futile. Other than catching COVID-19 from people you live with, in which case there's no need for contact tracing, it's impossible in most cases to figure out who you caught it from. Also, contact tracing is very labor intensive and expensive. The policy that contact tracing is essential was abandoned without any new data on its effectiveness, or any science-based rationale for the discontinuation. The talk was limited to vaccines, lockdowns, social distancing and face masks. Throughout 2020 , while the need for contact tracing was disappearing, there was a lot of hyperbole about asymptomatic carriers. The threat posed by asymptomatic carriers was used to justify face mask mandates. If asymptomatic carriers are such a big threat, though, what is the point of contact tracing? A person who developed symptoms of COVID-19 would have to notify authorities, who in turn would have to notify everyone that person had come in contact with during the previous week. But then we would have to test countless people to identify the asymptomatic carriers. This could include everyone who was at a grocery store at the same time as the symptomatic person. How would all these people be identified and notified? The whole thing was logistically impossible. The two social control strategies of contact tracing and ramping up fear of asymptomatic carriers contradicted each other. No problem - we just dropped one of them. Actually, we've pretty much dropped both of them - the amount of emphasis on asymptomatic carriers in the media has dwindled a lot in 2021.

The same thing happened with testing - quietly stopping any mention of it. Up to mid-2020 there was a big emphasis on the need for testing. This need could have been met with a series of randomized testing studies in representative areas of the country, but these were never conducted. Instead, there was scattered, inconsistent testing throughout the United States. The percentage of positive tests was used to track the pandemic, and there was a lot of emphasis on getting the percentage of tests positive down under 10\%. By mid-2021, public discussion about testing had pretty much vanished. Why? This doesn't make any scientific or epidemiological sense. Wouldn't we want to emphasize falling positivity rates to promote the idea that the vaccines are curbing the pandemic? Overall, recommendations about contact tracing and testing have fluctuated widely over the last 18 months, without any data being gathered or presented to the public to justify the changes. We just stopped talking about these two prior pillars of public health.

\section{Disputes about Herd Immunity}

If $100 \%$ of the population had SARS-CoV-2 antibodies this would result in herd immunity and stop the pandemic. This is true no matter what percentage of the immunity comes from vaccination and what percentage from natural infection. The medical profession has been unanimous on this point. What is the problem then?
The problem has been the politicization of discussion of herd immunity to attack and discredit physicians who emphasize the natural infection component of herd immunity. The need for herd immunity has been a major selling point for vaccines by the medical profession. Generally, 70\% herd immunity is regarded as the threshold goal. Anything beyond that is welcome, but anything less leaves us relatively unprotected. This is true. Emphasizing natural immunity has been equated with being anti-science and anti-vaccine. Why? If $40 \%$ of people get vaccinated and an additional $40 \%$ have natural immunity due to being infected, then we have reached meaningful herd immunity. It doesn't matter what the numbers are - the principle remains the same. Herd immunity is the sum of vaccine-derived immunity and infection-derived immunity. Unfortunately, we don't have an exact figure for the percentage of the population that has been infected, even though an accurate figure $+/$ - a small margin of error could be obtained by random sampling across the nation., at a low cost compared to the total financial burden of the pandemic.

Rather than attacking physicians who emphasize the natural immunity component of herd immunity, the public should be informed that in order to reach $70 \%$ herd immunity, fewer than $70 \%$ of people need to be vaccinated. Instead, the natural immunity component is not mentioned and is left out of the analysis. That may be fine as a motivational strategy for people to get vaccinated, but it is not science. Why can't we have a rational, data-based discussion of herd immunity, rather than polarized political warfare? I don't mean among politicians - I mean in the medical profession.

\section{Attributing Reductions in Infections and Deaths to the Vaccines}

Dropping rates of hospitalizations and deaths from COVID-19 in spring, 2021 have been widely attributed to the vaccines by the medical profession and public health officials. There is little mention of the fact that viral illness rates always go down in the summer. It could be that the vaccines are contributing but it could be that it is all due to the change in seasons. Most likely it is a combination of both. The percentage of the population vaccinated is far below the threshold for meaningful herd immunity, so it can't all be due to vaccines. A balanced, data-based, analytical discussion of the contributions to falling rates is not allowed because that might interfere with promoting vaccines. Again, this could be fine as a public health and population control strategy, but it is not science-based.

\section{Discounting the Social and Economic Costs of Lockdowns}

An analysis of the costs and benefits of any medical or public health intervention is a standard approach. The gold standard is randomized controlled trials (RCTs) - everyone in medicine agrees on that, even though RCTs are not always feasible or available. Interventions that cause substantial morbidity and mortality such as chemotherapy and some surgical procedures are nevertheless ethical to prescribe. If more people die without the intervention than die from it, it is good medicine to recommend the intervention, after an informed consent discussion with the patient. No one disputes this in medicine. Antivaxxers who are alarmed about cases of post-vaccine morbidity and mortality fail to understand the principle of cost-benefit analysis. 
They also fail to take into account the base rate of the morbidity and mortality in the general population. They are alarmists for no sound scientific reason.

The medical profession makes the opposite error. For example, they belittle anyone concerned about severe vaccine side effects but either ignore or are unaware of the fact that the government of the United States has awarded over $\$ 4,000,000,000.00$ due to vaccine damages. This has been done through the Vaccine Court - the National Vaccine Injury Compensation Program. This Court has a very high threshold of proof.

There are no other legal remedies for vaccine injuries because vaccine manufacturers and doctors are immune from liability for vaccine injuries. Vaccines are the only medical or public health intervention with such liability protection. Why is that?

The problem is not the desire to encourage people to get vaccinated. The problem is ignoring or distorting the science by making global statements that vaccines are 'safe and effective' - some years the flu vaccine is under $10 \%$ effective according to the CDC, whereas the measles and COVID-19 vaccines appear to be over $90 \%$ effective. Global statements that vaccines are 'safe and effective' are not based on science. The base rate, morbidity and mortality of a disease in the general population, and the effectiveness and adverse effects of a vaccine for it all have to be weighed in reaching an evidence-based decision. The Vaccine Adverse Event Reporting System (VAERS) currently has over 4,000 reported deaths related to COVID-19 vaccines, far more than the combined total for all other vaccines over a longer time period. When VAERS deaths from other vaccines are low, this is hailed as proof that the vaccines are safe. When they are high for COVID-19 vaccines, anyone who points this out is attacked and discredited as anti-science and an anti-vaxxer. The same is true for the costs of lockdown - economic, mental health, relationship, educational, on and on - not to mention opiate overdoses, murders, domestic violence, child abuse and blocked access to surgery, cancer treatment and other medical services. The medical profession and public health authorities have not provided a balanced cost-benefit analysis. Instead they have attacked and belittled anyone who emphasizes the cost side of the equation and have characterized them as not caring about grandma. Maybe the quality of grandma's last year of life was severely impacted by the lockdowns. The fact that lockdowns have substantial costs doesn't automatically mean they are bad. But ignoring the cost side of the equation is politics not science.

\section{If Trump is for it, We are against it}

If Trump is for it, we are against it. This theme has continued throughout the pandemic: whatever Trump says has to be attacked and discredited. Physicians who attacked Trump for politicizing the pandemic have been politicizing it themselves, but in the opposite direction. For them God is on the anti-Trump side. While doing so these physicians have adopted the pose of being science-based and have attacked people who disagreed with them as being unscientific. Actually, science is on neither side. An example is the once-ridiculous Trump conspiracy theory that the pandemic originated from a leak at the Wuhan Institute of Virology. This theory was savagely belittled as Trump nonsense. Now, Biden is being hailed for taking it seriously. The medical profession has been - by and large - marching to the same drummer. The further away we get from the Trump administration, the more easily his policies can be rehabilitated as Biden corrections to Trumpian excesses and errors - even when they are the same policies. All credit for vaccine rollout to Biden, none to Trump. Biden makes this claim because he is a politician - the medical profession should not agree with him. Credit should be given where credit is due; it should not be distributed as a political favor. The medical profession needs to get its house in order. It needs to be based, as much as possible, on data, science and rational analysis, not on partisan politics disguised as science.

\section{References}

1. Ross CA (2021) Misinformation concerning face masks and the Wuhan lab leak. Journal of Neurology Neurocritical Care 4: 1-3.

2. Ross CA (2020) Differences in evaluation of hydroxychloroquine and face masks for SARS-CoV-2. Journal of Neurology and Neurocritical Care 3: 1-3.

3. Ross CA (2020) Thoughts on COVID-19. Journal of Neurology and Neurocritical Care 3: 1-3.

4. Ross CA (2020) Facemasks are not effective for preventing transmission of the coronavirus. Journal of Neurology and Neurocritical Care 3: 1-2.

5. Ross CA (2020) How misinformation that facemasks are effective for reducing COVID-19 is transmitted. Journal of Neurology Neurocritical Care 3: 1-2.

6. Ross CA (2021) COVID Face masks and the Wuhan lab escape theory: An update. Journal of Neurology Neurocritical Care 4: 1-3. 